\title{
Guanidine $N$-methylation by BIsL Is Dependent on Acylation of Beta-amine Arginine in the Biosynthesis of Blasticidin S
}

\author{
Xiankun Wang, Aiqin Du, Guiyang Yu, Zixin Deng and Xinyi He* \\ State Key Laboratory of Microbial Metabolism, School of Life Sciences and Biotechnology, Shanghai Jiao Tong University, \\ Shanghai, China
}

The peptidyl nucleoside blasticidin S (BS) produced by Streptomyces griseochromogenes was the first non-mercurial fungicide used to prevent rice blast and increasingly used as a selection reagent in transgenic study. Acylation by addition of a leucine residue at the beta amine group of arginine side chain of demethylblasticidin $S$ (DBS) has been proposed as a novel self-resistance to the cytotoxic biosynthetic intermediate. But the resultant product leucyldemethylblasticidin S (LDBS) has not been isolated as a metabolite, and LDBS synthetase activity remained to be demonstrated in

OPEN ACCESS

Edited by:

Claudio Fabricio Gonzalez, University of Florida, United States

Reviewed by:

Youli Xiao,

Shanghai Institutes for Biological Sciences (CAS), China Wenqing Chen,

Wuhan University, China

${ }^{*}$ Correspondence:

Xinyi He

xyhe@sjtu.edu.cn

Specialty section:

This article was submitted to Microbial Physiology and Metabolism, a section of the journal

Frontiers in Microbiology

Received: 22 June 2017 Accepted: 02 August 2017

Published: 22 August 2017

Citation:

Wang $X$, Du A, Yu G, Deng Z and He X (2017) Guanidine N-methylation

by BIsL Is Dependent on Acylation of Beta-amine Arginine in the Biosynthesis of Blasticidin S.

Front. Microbiol. 8:1565.

doi: 10.3389/fmicb.2017.01565
S. griseochromogenes. In this study, we isolated LDBS in a BS heterologous producer S. lividans WJ2 upon the deletion of blsL, which encodes a S-Adenosyl methioninedependent methyltransferase. Purified BlsL efficiently methylated LDBS at the delta N of beta-arginine to generate the ultimate intermediate LBS, but nearly didn't methylate DBS to final product BS. Above experiments demonstrated that LDBS is indeed an intermediate in BS biosynthetic pathway, and acylation of beta-amino group of arginine side chain is prerequisite for efficient guanidine $\mathrm{N}$-methylation in addition to being a self-resistance mechanism.

Keywords: arginine $\mathrm{N}$-methyltransferase, blasticidin $\mathrm{S}$ biosynthesis, nucleoside resistance, leucyldemethylblasticidin $\mathrm{S}$, substrate preference

\section{INTRODUCTION}

Blasticidin S (BS) was first isolated from the broth of Streptomyces griseochromogenes as an effective non-mercurial fungicide (Takeuchi et al., 1958). BS displayed wide-range antimicrobial spectrum, it exerts its biological activities by inhibiting protein translation through a unique mechanism by which it traps the deformed P-site tRNA and strongly suppresses peptidyl-tRNA hydrolysis by release factors and peptide bond formation (Svidritskiy et al., 2013). As it's friendly to environment and not harmful to fish, blasticidin $\mathrm{S}$ was soon used on a large scale by foliar application in Asia to control rice blast caused by Pyricularia oryzae (Misato et al., 1958). Now it has been increasingly used as the selection reagent in mammalian transgenic studies (Kamakura et al., 1990; Freitas et al., 2002). Moreover, BS strongly inhibits the production of aflatoxin by fungus Aspergillus flavus that is toxic and highly carcinogenic (Yoshinari et al., 2013).

Blasticidin $\mathrm{S}$ is consisted of a $\mathrm{N}$-methyl-guanidine tail attached to a pyranose ring and bonded to a cytosine (Ōtake et al., 1966), its core moiety cytosylglucuronic acid (CGA) is shared by mildiomycin, gougerotin, arginomycin (Fox et al., 1968; Harada et al., 1978; Argoudelis et al., 1987). 
Unfortunately, studies on BS biosynthesis have proceeded slowly since the cloning of BS biosynthetic gene cluster as gene deletion in BS native producer S. griseochromogenes was never successful. In order to bypass the restriction barrier, we engrafted BS biosynthetic gene cluster onto the chromosome of S. lividans and generated a BS heterologous producer S. lividans WJ2 (Li et al., 2013), which here was used for in vivo study.

The putative BS biosynthetic pathway was proposed based on feeding experiments (Seto et al., 1968) and determination of metabolites (Prabhakaran et al., 1988; Guo and Gould, 1991; Gould and Zhang, 1995). However, several steps and their catalyzed enzymes remain to be established. A most intriguing question regarding BS biosynthesis is BS maturation. Demethylblasticidin S (DBS) had been isolated as a minor metabolite in the broth of $S$. griseochromogenes, and was proposed as the penultimate metabolite for $N$-methylation to give the final BS. But in the presence of S-Adenosyl methionine (SAM), the CFE of $S$. griseochromogenes could only methylate DBS at a ratio of as low as $0.85 \%$. In contrast, a derivative named AcDBS, with the beta amino group of guanidine acetylated, could be methylated to form AcBS at a ratio of 21\% (Zhang et al., 2000). However, AcBS cannot be hydrolyzed by the CFE of $S$. griseochromogenes to form mature BS, therefore both DBS and AcDBS were excluded as the direct precursors for $N$-guanidine methylation. On the other hand, leucylblasticidin $\mathrm{S}$ (LBS) was sometimes isolated as an intermediate in the broth of $S$. griseochromogenes when the $\mathrm{pH}$ of the culture medium was kept below 4 (Seto et al., 1968), it was later isolated from Streptomyces sp. SCC 1785 (Cooper et al., 1988) and S. griseochromogenes under normal laboratory conditions (Zhang et al., 2000). Discovery of LBS as the metabolite implied that the real intermediate for $N$-methylation might be LDBS rather than DBS. Interestingly, chemically synthesized LDBS displayed 20-fold less inhibition activity against Bacillus circulans than DBS. Therefore, leucylation of DBS into LDBS was postulated to be a novel self-resistance mechanism to protect the host from the toxic biosynthetic intermediate. However, the intermediacy of LDBS in BS biosynthesis remains elusive because LDBS is rarely detected in the native producer $S$. griseochromogenes, and the attempts to demonstrate LDBS synthetase activity were not successful (Zhang et al., 2000).

Here, we isolated LDBS as a major metabolite upon the deletion of the SAM-dependent guanidine methyltransferase gene $b l s L$ in a BS heterologous producer S. lividans WJ2. Moreover, BlsL can efficiently methylate LDBS into LBS, similar to methylation of the acetyl-DBS, but nearly cannot methylate DBS, demonstrating acylation of beta-amine is prerequisite for efficient methylation of guanidine $N$-methylation in BS biosynthesis beyond being as a self-resistance mechanism.

\section{MATERIALS AND METHODS}

Compound Blasticidin S was purchased from YEASEN Biotech Co., Ltd. (Shanghai, China). DBS, LDBS, and LBS were synthesized by Professor Mark Zabriskie at Oregon State University (Zhang et al., 2000). SAM, L-arabinose, apramycin, spectinomycin, kanamycin and trimethoprim (TMP) were purchased from Sangon Biotech Co., Ltd. (Shanghai, China). S. lividans WJ2 contains the BS biosynthetic gene cluster and capable of producing blasticidin S (Li et al., 2013), S. lividans WXK1 is derived from WJ2 by deletion of blsL gene, S. lividans WXK2 is derived from WXK1 complemented with $b l s L$ in an integrative plasmid pIB139. All Streptomyces strains (Supplementary Table S1) were cultivated at $30^{\circ} \mathrm{C}$ on Soy Flour-Mannitol (SFM) agar plates for sporulation, mycelia were grown in $10.3 \%$ TSBY (tryptic soy broth supplemented with $10.3 \%$ sucrose (wt/vol) and $1 \%$ yeast extract (wt/vol) medium. E. coli strain $\mathrm{DH} 10 \mathrm{~B}$ was used for construction cloning vectors, BW25113/pIJ790 was used for in-frame deletion of blsL gene by PCR targeting, ET12567/pUZ8002 was used for conjugal transfer with Streptomyces. E. coli BL21(DE3) (Novagen) was used as a host for protein expression. E. coli strains (Supplementary Table S1) were cultivated at $37^{\circ} \mathrm{C}$ in LB/LA medium containing corresponding antibiotic. Plasmid pJTU1780 contains $35 \mathrm{~kb}$ fragment of BS biosynthetic cluster and pET28a (Novagen) is for protein overexpression.

\section{General Molecular Biology Methods}

Plasmid DNA were purified by plasmid mini kit (Omega), isolation of genomic DNA and other manipulations of Streptomyces were performed using the method described by Keiser et al. (2000). Restriction enzymes NdeI, EcoRI and HindIII and DNA polymerase were purchased from New England Biolabs (Beijing) Ltd. Primers (Supplementary Table S1) were synthesized by JIE LI BIOLOGY (Shanghai, China), PCR products were purified from agarose gels $(0.8 \%)$ using DNA Gel Extraction Kit (Omega). T4 ligase kit for construction of recombinant plasmid was purchased from TaKaRa (China). The PCR-targeting method for in-frame deletion of blsL was done according to Bertolt Gust (Gust et al., 2002).

\section{HPLC and LC-MS Analysis}

The acquisition of the fermentation products of Streptomyces lividans WJ2 and its derived strains were employed using secondary fermentation according to Li et al. (2013). After 2-6 days of fermentation, $25 \mathrm{ml}$ fermentation broth was transferred to a $50 \mathrm{ml}$ centrifuge tubes, $5000 \mathrm{r} / \mathrm{min}$ for $10 \mathrm{~min}$, the supernatant was collected and adjusted to $\mathrm{pH} 5.0$ using oxalic acid and then lay aside at room temperature for $30 \mathrm{~min}$ before centrifugation (10000 r/min, $30 \mathrm{~min})$ again. The Supelclean LC-SCX solid-phase extraction (SPE) columns (bed weight, $500 \mathrm{mg}$; volume, $3 \mathrm{ml}$ ) (Supelco) was used for purification of BS and other intermediates. The column was activated by washing with $3 \mathrm{ml}$ methanol and $5 \mathrm{ml}$ sterile water, the supernatants were injected to penetrate the column and followed by injection of $5 \mathrm{ml}$ sterile water to remove unwanted inclusions, then $5 \mathrm{ml}$ $0.5 \%$ ammonia solution were flowed through the column to further remove impurities. Finally, $3 \mathrm{ml} 5 \%$ ammonia solution were used to elute BS-related products. HPLC analysis was performed on an Agilent series 1260 using the innoval C18 column (4.6 mm $\times 250 \mathrm{~mm}$, Agela Technologies). Two buffer were used, buffer A1: 0.1\% (vol/vol) trifluoroacetic acid in water was aqueous phase, buffer B was organic phase (methanol). 
The samples were eluted at a flow rate of $0.3 \mathrm{ml} / \mathrm{min}$ and the concentration of buffer B rose from 5 to $40 \%$ in $40 \mathrm{~min}$. The substances were detected at UV275. To confirm the molecular weight of each peak, a LC-MS analysis was done using an Agilent 1100 LC/MSD (mass-selective detector) with electrospray ionization (ESI) in the positive mode.

\section{Overexpression and Purification of BIsL}

High-fidelity DNA polymerase Hieff TM Pfu (YEASEN BiotechCo., Ltd., Shanghai, China) was used to amplify the blsL gene sequence from plasmid pJTU1780 with the Forward primer: GGAATTCCATATGGAGCAGAGCACCGGCGCGAT and Reverse primer: CGGAATTCTCAGTCCTTGTAGAAACACT (NdeI and EcoRI sites are underlined) by PCR. The PCR product were purified and digested by NdeI and EcoRI and ligated with pET28a vector which was also cut by NdeI and EcoRI, the ligation mixture was transformed into E. coli DH10B. After confirmation of the right clone by sequencing, recombinant plasmid pET28a-blsL was transformed into E. coli BL21(DE3) and grown overnight at $37^{\circ} \mathrm{C}$ in $10 \mathrm{ml} \mathrm{LB}$ medium supplied kanamycin (final concentration $50 \mu \mathrm{g} / \mathrm{ml}$ ). $10 \mathrm{ml}$ of cell culture were inoculated in $1 \mathrm{~L} \mathrm{LB}$ medium in a $3 \mathrm{~L}$ flask and cultured at $37^{\circ} \mathrm{C}$ until OD600 approaching 0.6 and then $250 \mu \mathrm{l}$ isopropylD-thiogalactopyranoside (IPTG, $800 \mathrm{mM}$ ) was added to a final concentration of $0.2 \mathrm{mM}$, the culture medium was then transferred to $16^{\circ} \mathrm{C}$ and grown for $16 \mathrm{~h}$. Cells were harvested by centrifugation at $5000 \mathrm{r} / \mathrm{min}$ for $10 \mathrm{~min}$ and suspended in $50 \mathrm{ml}$ of lysis buffer (50 mM Tris- $\mathrm{HCl} \mathrm{pH} \mathrm{7.8,0.5} \mathrm{M} \mathrm{NaCl}$ and $20 \mathrm{mM}$ imidazole) and lysed by high power press cell disrupter (UH-240/UH-480/UH-960, Union-Biotech, Co., Ltd.) at 800 bar for $5 \mathrm{~min}$. Then centrifugation at $10000 \mathrm{r} / \mathrm{min}$ for $1 \mathrm{~h}$ and the supernatant were applied to a column filled with $3 \mathrm{ml}$ Ni-NTA agarose (GE Healthcare), washing the column with $60 \mathrm{ml}$ washing buffer $(50 \mathrm{mM}$ Tris- $\mathrm{HCl} \mathrm{pH} \mathrm{7.8,} 0.5 \mathrm{M} \mathrm{NaCl}$ and $40 \mathrm{mM}$ imidazole) and eluted the His-tagged protein $\mathrm{BlsL}$ with $15 \mathrm{ml}$ elution buffer (50 mM Tris- $\mathrm{HCl}$ pH 7.8, $0.5 \mathrm{M} \mathrm{NaCl}$ and $300 \mathrm{mM}$ imidazole). The eluted BlsL was desalted using PD10 desalting column (GE Healthcare) and stored in storing buffer (25 mM Tris- $\mathrm{HCl}$ buffer $\mathrm{pH} 7.8,100 \mathrm{mM} \mathrm{NaCl}$ and 10\% glycerol). The purity of BlsL was detected by SDS-PAGE and the concentration of BlsL was determined by UV absorption at A280 using NanoDrop (Thermo).

\section{In Vitro Assay of the Purified BIsL}

The in vitro assay of BlsL was carried out in reaction buffer (25 mM Tris- $\mathrm{HCl}$ buffer, $\mathrm{pH} 7.8$ and $100 \mathrm{mM} \mathrm{NaCl}$ ) at $37^{\circ} \mathrm{C}$ for $1 \mathrm{~h}$ or overnight, the reaction volume was $100 \mu \mathrm{l}$ with $10 \mu \mathrm{M}$ BlsL, $1 \mathrm{mM}$ DBS or $1 \mathrm{mM}$ LDBS and $2 \mathrm{mM}$ SAM. The reactions were quenched by adding equal volume of chloroform and shocked for $1 \mathrm{~min}$ to denature the BlsL and the supernatant was collected by centrifugation $(12000 \mathrm{r} / \mathrm{min}, 10 \mathrm{~min})$. The measurement of the reaction products was performed by HPLC and LC-MS using TC-C18 (4.6 mm × $250 \mathrm{~mm}$, Agilent). Aqueous phase was buffer A2 (20 mM aqueous phase, $\mathrm{pH} 5.5$ ), organic phase was still buffer $B$, the sample were eluted at a flow rate of $0.3 \mathrm{ml} / \mathrm{min}$ with buffer B rising from 10 to $50 \%$ in $30 \mathrm{~min}$.

\section{RESULTS}

\section{Analysis of BIsL, a SAM-Coordinated Methyltransferase in Blasticidin S Biosynthesis}

$b l s L$ is the sole methyltransferase gene in BS biosynthetic gene cluster, and thus proposed to be in charge of Guanidine$\mathrm{N}$-methylation. Sequence analysis of BlsL revealed that low similarity to guanidine acetate methyltransferase (GAMT, 33\% identity, $85 \%$ similarity) and protein arginine methyltransferase (PRMT, 35\% identity, 74\% similarity). GAMT catalyzes guanidinoacetate (GAA) and SAM into creatine and S-adenosylhomocysteine (SAH). GAMT deficiency causes mental retardation, speech delay, and epilepsy (Velichkova and Himo, 2006). PRMT both catalyzes the formation of monomethylarginine (MMA) and symmetrical dimethylarginine (SDMA) from arginine residues of versatile proteins, and is involved in regulation of many cellular processes (Poulard et al., 2016). Both GAMT and PRMT catalyze $N$-methylation of guanidine group, but differs in the selection of target $\mathrm{N}$ atoms (Figure 1).

Sequence comparison revealed that BlsL contains a SAMcoordination motif E/DXGXGXGX that is conserve in above two protein families (Supplementary Figure S1) (Martin and McMillan, 2002). Corresponding to this, free SAM can be released from boiled BlsL that was heterologously expressed and purified in E. coli. Association of SAM with BlsL was confirmed by HPLC as well as MS/MS fragmentation (Supplementary Figure S2).

\section{LDBS Is Isolated As an Intermediate upon Deletion of b/sL in a Blasticidin S Heterologous Producer}

As genetic manipulation in BS native producer S. griseochromogenes has been not successful. We studied BS biosynthesis in a heterologous producer S. lividans WJ2 (Li et al., 2013). In order to know the role of BlsL in the biosynthesis of $\mathrm{BS}, b l s L$ was in-frame deleted in WJ2 to generate mutant WXK1. Compared with WJ2, two peaks in HPLC assay corresponding to LBS and BS disappeared in the fermentation profile of mutant WXK1 whereas the demethylated intermediates DBS and LDBS were retained and yields of them were significantly enhanced (Figure 2). Complementation of WXK1 with blsL in trans under constitutive promoter ermE* on integrative plasmid pIB139 can restore the mutant production of LBS and BS. These demonstrating that BlsL is the only enzyme in WJ2 governing delta- $\mathrm{N}$ methylation of guanidine side chain, the detection of LDBS, especially in mutant WXK1 implied that LDBS is a true intermediate for BS biosynthesis.

\section{BIsL Effectively Catalyzes Guanidine $\mathrm{N}$-methylation of LDBS}

The co-existence of DBS and LDBS in fermentation broth of both WJ2 and WXK1 made it hard to know which one is the true substrate for BlsL. In order to address this question, 
<smiles>N=C(N)NC(=O)O</smiles>

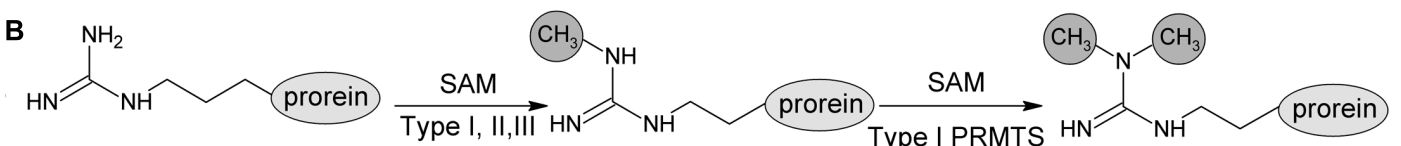
Arginine residue $\quad$ PRMTS Monomethylarginine(MMA)

C<smiles>N=C(N)N(CC[C@H](N)CC(=O)N[C@H]1C=CC(n2ccc(N)nc2=O)OC1C(=O)O)c1ccccc1</smiles>

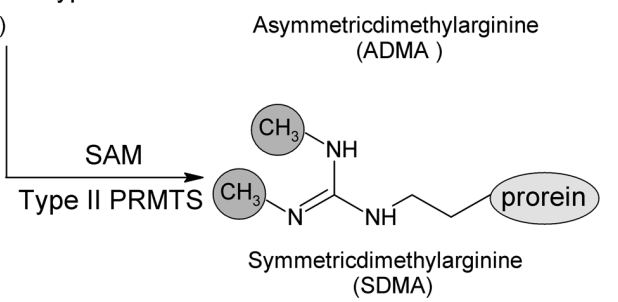

FIGURE 1 | Methyl-transfer reaction catalyzed by GMAT and PRMTs. (A) Guanidino acetate methyltransferase (GAMT) catalyzes the guanidinoacetate (GAA) and S-adenosyl methionine (SAM) into creatine and S-adenosylhomocysteine (SAH). (B) Protein arginine methyltransferase (PRMT) family containing three types (Types I, II and III) according to the type of methylation they catalyze. All of these types produce $\omega$-NG-monomethylarginine (MMA); additionally, Type I PRMTs are responsible for producing $\omega$-NG-NG-asymmetric dimethylarginine (ADMA) and Type II PRMTs are for producing $\omega$-NG-N'G-symmetric dimethylarginine (SDMA). (C) Methyl group at delta $\mathrm{N}$ of beta-amine arginine of blasticidin $\mathrm{S}$ is catalyzed by BlsL.

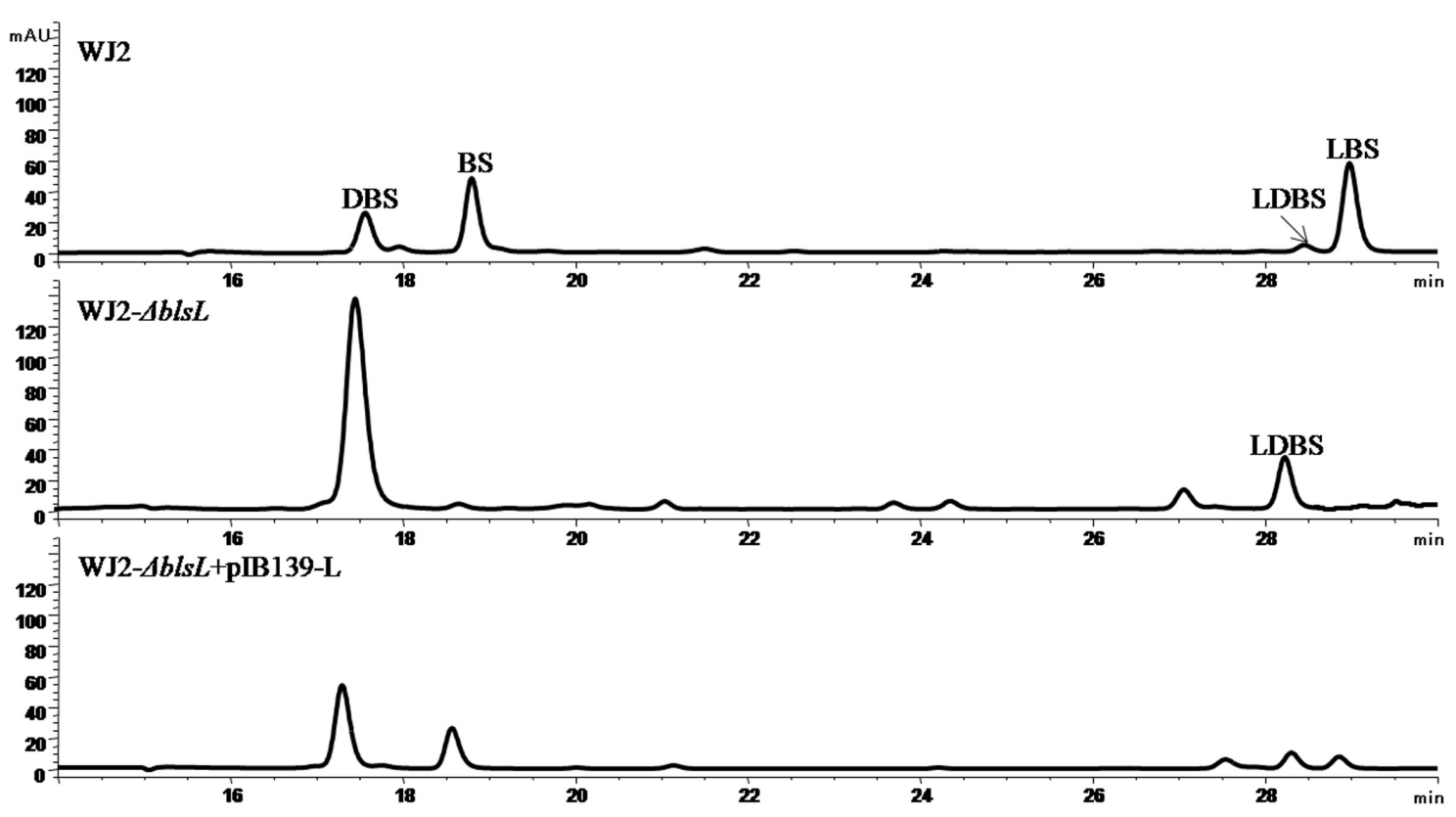

FIGURE 2 | HPLC analysis of fermentation products of BS heterologous producer strain S. lividans WJ2 and two mutant strains WJ2 ( $\Delta$ blsL) and WJ2 ( $\triangle$ b/sL)::b/sL. The column used for analysis of metabolites here is Innoval C18 column (4.6 mm $\times 250 \mathrm{~mm}$, Agela Technologies), and the elution conditions please refer to the Section "Materials and Methods."

we measured the methyltransferase activity of purified BlsL on chemically synthesized LDBS and DBS (Gifts from Prof. Mark Zabriskie) (Zhang et al., 2000). Compared to the boiled BlsL, purified BlsL (Figure 3A) can convert $80 \%$ of LDBS into LBS in 1-h incubation; concomitantly, $90 \%$ of SAM were consumed and turned into SAH (Figure 3B). We also noticed that the control reaction without SAM also gave 5\% conversion of LDBS into LBS, whose structure was confirmed by secondary MS fragmentation (Figure 3C), consistently supporting that purified BlsL contains SAM and catalyzes the $N$-methylation of LDBS.

\section{BIsL Nearly Doesn't Methylate DBS}

In sharp contrast, two substrates DBS and SAM both kept intact after 1 -h reaction with BlsL, and there is no visible peak in HPLC 

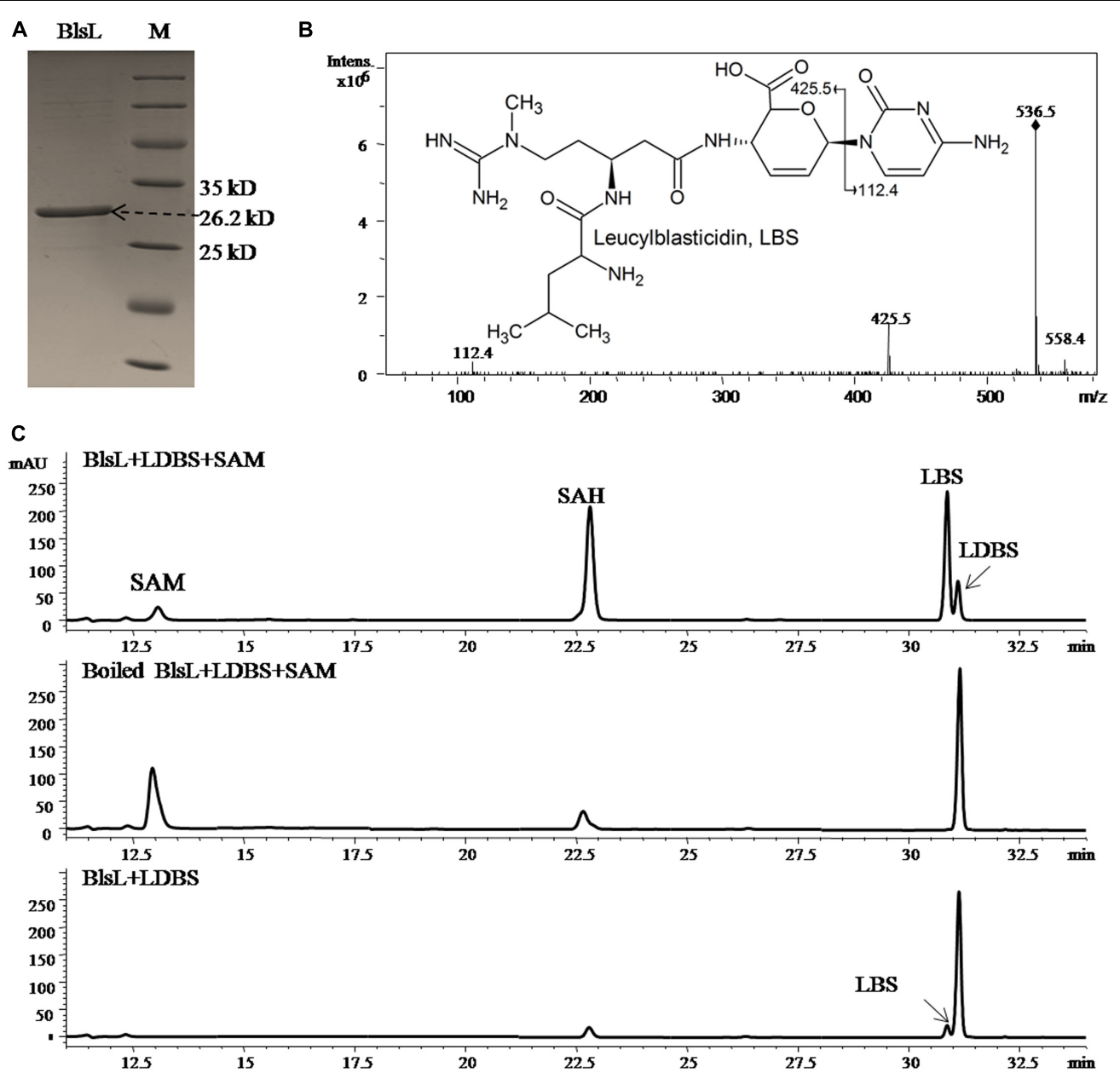

FIGURE 3 | BIsL efficiently catalyses the formation of LBS with LDBS as the substrate. (A) SDS-PAGE analysis of purified BlsL; (B) MS/MS fragments of LBS generated by BlsL-catalyzed methylation with LDBS and SAM, (C) HPLC analysis of the reaction products of BlsL with LDBS and SAM. The measurement of the reaction products were performed by HPLC and LC-MS using TC-C18 (4.6 mm $\times 250 \mathrm{~mm}$, Agilent) and the elution conditions please refer to the Section "Materials and Methods."

corresponding to BS, for which DBS was previously proposed to be the direct substrate (Figure 4A). These results demonstrated that DBS cannot be catalyzed by BlsL. However, MS spectrum analysis detected the ion at M/Z 423 that corresponds to the mass for BS (Figure 4B), secondary MS fragmentation pattern further supported the formation of BS (Figure 4C), consistent with the result that only $0.85 \%$ of DBS can be converted to BS by the cell of S. griseochromogenes (Zhang et al., 2000).

Taken together, the last four biosynthetic steps are summarized as in Figure 5. Namely, DBS can nearly not be directly converted into BS whereas LDBS is the true substrate for $\mathrm{BlsL}$, and thus demonstrating that LDBS is the real intermediate for methylation in BS biosynthesis.

\section{DISCUSSION}

In the study of BS biosynthesis, Seto and Yonehara (1977) isolated intermediate DBS and a minor metabolite LBS in S. griseochromogenes. Both compounds require a single transformation for conversion to BS (Figure 5). DBS was assayed as the precursor for BS by using the Cell-Free-Extract of S. griseochromogenes, but the SAM and DBS both were not consumed. So DBS is not the direct precursor for the guandino methylase. In the light of biosynthetic study of another nucleoside puromycin, the $2^{\prime \prime}$-amino group of $O$-methyl-L-tyrosine moiety was acetylated in the presence of acetyl coenzyme A, and was followed by methylation (Pérez-González et al., 1983; Sugiyama et al., 1985).

The compound acetyl-DBS (AcDBS) with the beta-amino group of arginine side chain by acetylated was assayed and found to be efficiently converted to AcBS, implied that acetylation of beta-arginine is essential to the guanidine $N$-methylation. However, AcDBS couldn't be hydrolyzed into mature BS, and thus is not the biosynthetic intermediate for BS.

The switching relationship between two metabolite DBS and LBS requires leucylation of DBS to form LDBS followed by methylation of guanidine group (Figure 5). Chemically 
A

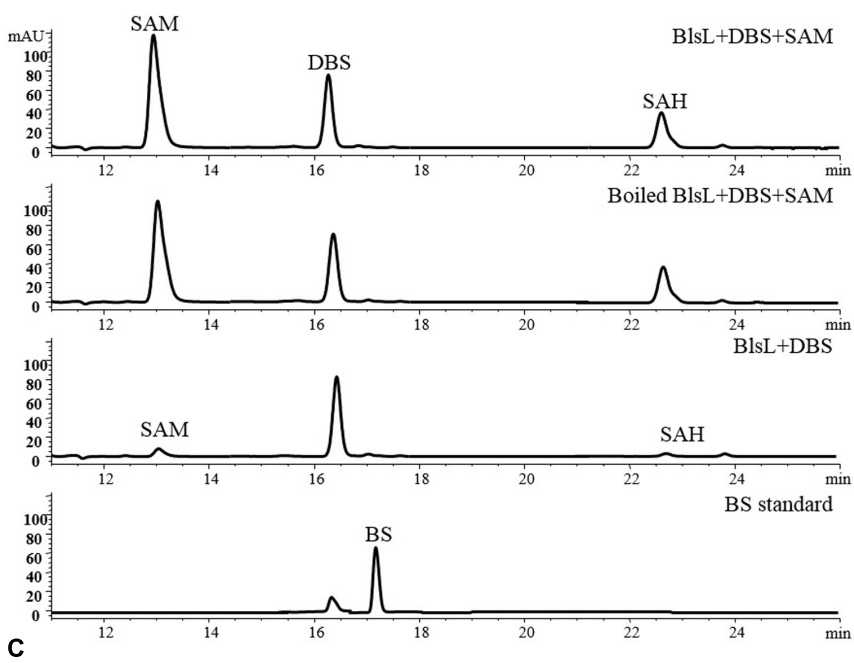

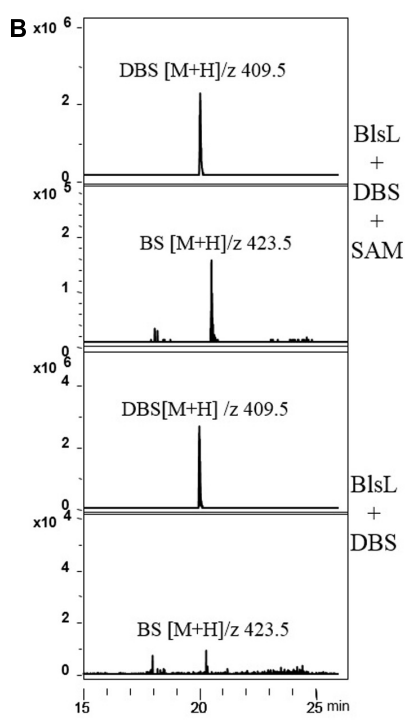

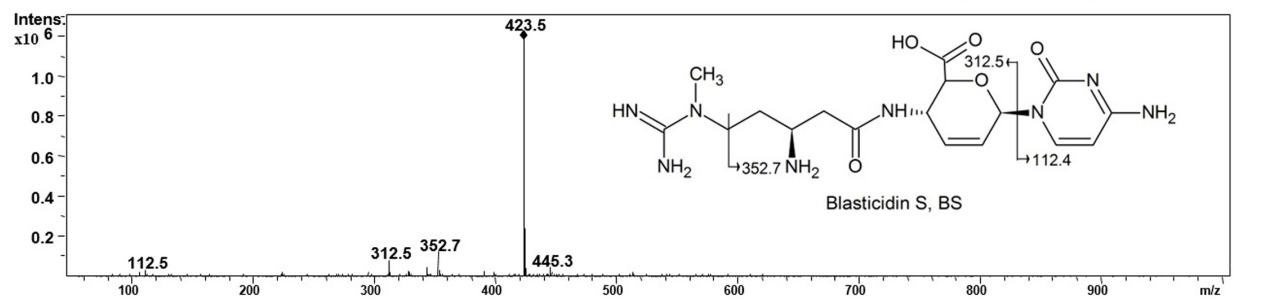

FIGURE 4 | BIsL nearly cannot catalyze the formation of BS with DBS as the substrate. (A) HPLC analysis of the reaction products of BlsL with DBS and SAM; (B) Increased intensity of ion for BS detected in the reaction of BlsL, DBS and SAM compared with boiled control protein; (C) MS/MS fragments of BS generated by BIsL-catalyzed methylation with DBS and SAM.

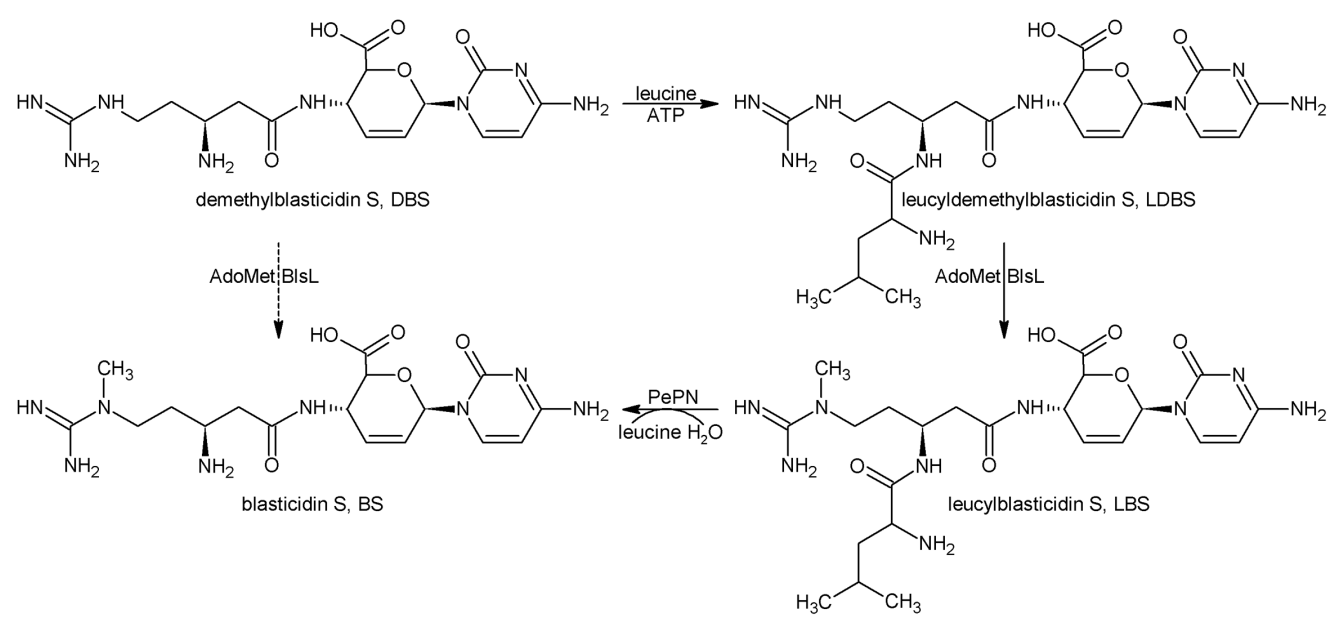

FIGURE 5 | The last four biosynthetic steps for BS. DBS, Demethylblasticidin S; LDBS, leucyldemethylblasticidin; LBS, leucylblasticidin S; BS, blasticidin S.

synthesized LDBS showed decreased cytotoxicity by 20 -folds compared to DBS, and thus leucylation of DBS was regarded as a novel self-resistance because of inactivation of an antibiotic with an amino acid (Zhang et al., 2000). This proposal was challenged by the failures both in isolations of intermediate LDBS and confirmation of LDBS synthetase activity in S. griseochromogenes.
In view of present situation of BS biosynthesis, failure in isolation of LDBS and only accumulation of very minor of LBS in the broth of S. griseochromogenes are attributed to efficient excision of leucine group from above two metabolites by standalone PepN and its homologs (Yu et al., 2015). Different from in the BS native producer, LBS is often presented as the dominant metabolite in 
a heterologous producer S. lividans WJ2, particularly when the $\mathrm{pH}$ of broth was kept below 6, this case usually occurred when the medium is contaminated in later phase of fermentation by acid-producing bacteria, such as E. coli. Also, LDBS could also be detected in WJ2, its production was apparently enhanced when the methyltransferase gene blsL was deleted (Figure 2). Furthermore, purified BlsL in vitro can effectively catalyze LDBS into LBS in the presence of SAM, but not methylate DBS, thus demonstrating the intermediacy of LDBS in BS biosynthesis.

Acylation of DBS at the beta-amine including AcDBS as well as LDBS significantly increased the methylation efficiency by BlsL S. griseochromogenes, implying that acylation of beta-amine might change the distribution of the charge or provide better fit each other between BlsL and the substrate either in recognition or catalysis. This work provided a solid base for demonstration of last four biosynthesis steps in BS biosynthesis.

\section{AUTHOR CONTRIBUTIONS}

$\mathrm{XW}$ and $\mathrm{AD}$ performed experiments. GY provided compounds and strains. $\mathrm{XW}, \mathrm{XH}$, and $\mathrm{ZD}$ analyzed the data. $\mathrm{XH}$ wrote the

\section{REFERENCES}

Argoudelis, A. D., Baczynskyj, L., Laborde, A., Sebek, O., Truesdell, S., and Shilliday, F. (1987). Arginomycin: production, isolation, characterization and structure. J. Antibiot. 40, 750-760. doi: 10.7164/antibiotics. 40.750

Cooper, R., Conover, M., and Patel, M. (1988). Sch 36605, structure of a novel nucleoside. J. Antibiot. 41, 123-125. doi: 10.7164/antibiotics. 41.123

Fox, J. J., Kuwada, Y., and Watanabe, K. A. (1968). Nucleosides LVI. On the structure of the nucleoside antibiotic, gougergtin. Tetrahedron Lett. 9, 6029-6032. doi: 10.1016/S0040-4039(00)70784-2

Freitas, A. C., Bento, F. M., Ramesh, N., Osborne, W. R., and Han, S. W. (2002). Modified blasticidin S resistance gene (bsrm) as a selectable marker for construction of retroviral vectors. J. Biotechnol. 95, 57-62. doi: 10.1016/S01681656(01)00442-4

Gould, S. J., and Zhang, Q. (1995). Cytosinine: pyridoxal phosphate tautomerase, a new enzyme in the blasticidin S biosynthetic pathway. J. Antibiot. 48, 652-656. doi: 10.7164 /antibiotics. 48.652

Guo, J., and Gould, S. J. (1991). Biosynthesis of blasticidin S from cytosylglucuronic acid (CGA). Isolation of cytosine/UDPglucuronosyltransferase and incorporation of CGA by Streptomyces griseochromogenes. J. Am. Chem. Soc. 113, 5898-5899. doi: 10.1021/ja00015a074

Gust, B., Kieser, T., and Chater, K. (2002). PCR Targeting System in Streptomyces coelicolor A3(2). Norwich: John Innes Centre.

Harada, S., Mizuta, E., and Kishi, T. (1978). Structure of mildiomycin, a new antifungal nucleoside antibiotic. J. Am. Chem. Soc. 100, 4895-4897. doi: $10.1021 /$ ja00483a047

Kamakura, T., Yoneyama, K., and Yamaguchi, I. (1990). Expression of the blasticidin S deaminase gene (bsr) in tobacco: fungicide tolerance and a new selective marker for transgenic plants. Mol. Gen. Genet. 223, 332-334. doi: 10.1007/BF00265072

Keiser, T., Bibb, M., Buttner, M., Chater, K., and Hopwood, D. (2000). Practical Streptomyces Genetics. Norwich: The John Innes Foundation.

Li, L., Wu, J., Deng, Z., Zabriskie, T. M., and He, X. (2013). Streptomyces lividans blasticidin $\mathrm{S}$ deaminase and its application in engineering a blasticidin S-producing strain for ease of genetic manipulation. Appl. Environ. Microbiol. 79, 2349-2357. doi: 10.1128/AEM.03 254-12 manuscript. All authors reviewed, revised, commented on and approved the final version of the manuscript.

\section{FUNDING}

This work received support from the National Natural Science Foundation of China (31470195).

\section{ACKNOWLEDGMENT}

We are grateful to Professor Mark Zabriskie for providing compounds LDBS and DBS.

\section{SUPPLEMENTARY MATERIAL}

The Supplementary Material for this article can be found online at: http://journal.frontiersin.org/article/10.3389/fmicb. 2017.01565/full\#supplementary-material

Martin, J. L., and McMillan, F. M. (2002). SAM (dependent) I AM: the S-adenosylmethionine-dependent methyltransferase fold. Curr. Opin. Struct. Biol. 12, 783-793. doi: 10.1016/S0959-440X(02)00391-3

Misato, T., Ishii, I., Asakawa, M., and Fukunaga, K. (1958). Antibiotics as protectant fungicides against rice blast. Jpn. J. Phytopathol. 23, 219-224. doi: 10.3186/jjphytopath.23.219

Ōtake, N., Takeuchi, S., Endō, T., and Yonehara, H. (1966). Chemical studies on blasticidin S. Agric. Biol. Chem. 30, 126-141. doi: 10.1080/00021369.1966. 10858563

Pérez-González, J. A., Vara, J., and Jiménez, A. (1983). Acetylation of puromycin by Streptomyces, alboniger the producing organism. Biochem. Biophys. Res. Commun. 113, 772-777. doi: 10.1016/0006-291X(83)91066-5

Poulard, C., Corbo, L., and Le Romancer, M. (2016). Protein arginine methylation/demethylation and cancer. Oncotarget 7, 67532-67550. doi: 10.18632/oncotarget.11376

Prabhakaran, P. C., Woo, N. T., Yorgey, P. S., and Gould, S. J. (1988). Biosynthesis of blasticidin S from L-. alpha.-arginine. Stereochemistry in the arginine-2, 3-aminomutase reaction. J. Am. Chem. Soc. 110, 5785-5791. doi: 10.1021/ ja00225a033

Seto, H., Otake, N., and Yonehara, H. (1968). Studies on the biosynthesis of blasticidin S. Agric. Biol. Chem. 32, 1299-1305. doi: 10.1271/bbb1961.32. 1299

Seto, H., and Yonehara, H. (1977). Studies on the biosynthesis of blasticidin S. VII. Isolation of demethylblasticidin S. J. Antibiot. 30, 1022-1024. doi: 10.7164/ antibiotics.30.1022

Sugiyama, M., Paik, S. Y., and Nomi, R. (1985). Mechanism of self-protection in a puromycin-producing micro-organism. Microbiology 131, 1999-2005. doi: 10.1099/00221287-131-8-1999

Svidritskiy, E., Ling, C., Ermolenko, D. N., and Korostelev, A. A. (2013). Blasticidin $S$ inhibits translation by trapping deformed tRNA on the ribosome. Proc. Natl. Acad. Sci. U.S.A. 110, 12283-12288. doi: 10.1073/pnas.1304922110

Takeuchi, S., Hirayama, K., Ueda, K., Sakai, H., and Yonehara, H. (1958). Blasticidin S, a new antibiotic. J. Antibiot. 11, 1-5.

Velichkova, P., and Himo, F. (2006). Theoretical study of the methyl transfer in guanidinoacetate methyltransferase. J. Phys. Chem. B 110, 16-19. doi: 10.1021/ jp055120d

Yoshinari, T., Sakuda, S., Watanabe, M., Kamata, Y., Ohnishi, T., and SugitaKonishi, Y. (2013). New metabolic pathway for converting blasticidin S in Aspergillus flavus and inhibitory activity of aflatoxin production by 
blasticidin S metabolites. J. Agric. Food Chem. 61, 7925-7931. doi: 10.1021/jf40 $2745 \mathrm{c}$

Yu, G., Li, L., Liu, X., Liu, G., Deng, Z., Zabriskie, M. T., et al. (2015). The standalone aminopeptidase PepN catalyzes the maturation of blasticidin S from leucylblasticidin S. Sci. Rep. 5:17641. doi: 10.1038/srep 17641

Zhang, Q., Cone, M. C., Gould, S. J., and Mark Zabriskie, T. (2000). Reevaluation of the final steps in the biosynthesis of blasticidin $\mathrm{S}$ by Streptomyces griseochromogenes and identification of a novel self-resistance mechanism. Tetrahedron 56, 693-701. doi: 10.1016/S0040-4020(99)01060-1
Conflict of Interest Statement: The authors declare that the research was conducted in the absence of any commercial or financial relationships that could be construed as a potential conflict of interest.

Copyright (c) 2017 Wang, Du, Yu, Deng and He. This is an open-access article distributed under the terms of the Creative Commons Attribution License (CC BY). The use, distribution or reproduction in other forums is permitted, provided the original author(s) or licensor are credited and that the original publication in this journal is cited, in accordance with accepted academic practice. No use, distribution or reproduction is permitted which does not comply with these terms. 